\title{
Evidenzbasierte Therapie der Rhinosinusitis
}

\section{So werden Nebenhöhlen wieder frei}

\author{
Ralph Mösges, Christine Heubach
}

\author{
Die S2-Leitlinie der Deutschen Gesellschaft für Hals-Nasen-Ohren-Heilkunde, Kopf- und \\ Hals-Chirurgie wird derzeit aktualisiert. Dieser Beitrag gibt einen Überblick über die \\ wichtigsten Aspekte zur Behandlung der Rhinosinusitis und berücksichtigt die jüngsten \\ Studienergebnisse.
}

D as Krankheitsbild der Rhinosinusitis wird nach der klinischen Symptomatik eingeteilt. Die Rhinosinusitis ist eine Entzündung der Nasennebenhöhlen mit mindestens zwei der folgenden Symptome:

- nasale Obstruktion

- Nasensekretion

- Gesichtsschmerz

— Druckgefühl

- Riechminderung.

Ferner sollten rhinoskopische Zeichen wie eitriger Ausfluss oder Schwellung bzw. Schleimhautobstruktion im mittleren $\mathrm{Na}$ sengang vorliegen.

Besteht die Symptomatik kürzer als zwölf Wochen und bilden sich die Beschwerden wieder vollständig zurück, liegt eine akute Rhinosinusitis viraler oder bakterieller Genese vor. Die Unter- scheidung zwischen einer viralen und einer bakteriellen Infektion ist anhand der klinischen Symptomatik schwierig. Nur bei schweren oder biphasischen Verläufen, Symptomzunahme nach fünf Tagen, Symptomdauer über mehr als zehn Tage oder beim Auftreten von Komplikationen geht man von einer bakteriellen Infektion aus.

Die chronische Rhinosinusitis liegt per Definition dann vor, wenn die Symptome länger als zwölf Wochen andauern, ohne sich zwischenzeitlichen vollständig zurückzubilden. Die Symptomatik ist weniger deutlich ausgeprägt; die Nasenatmung ist behindert, es bestehen Kopfschmerzen, Druckgefühl, Riechstörung und Nasensekretion; die allgemeine Infektanfälligkeit ist erhöht und die Patienten klagen über Erschöpfung.

\section{Umfrage zur Auswahl von Erkältungsmittel}

Für den "Erkältungstatus 2010", so der Titel einer von forsa durchgeführten und von STADA unterstützten Umfrage, wurden 76 HNO-Ärzte, 75 Allgemeinmediziner und 50 Apotheker per computergestützter Telefoninterviews befragt, welche Faktoren ihre Auswahl von Erkältungsmitteln bzw. entsprechende Empfehlungen beeinflussen.

Bei Entzündungen der oberen Atemwege ist sowohl für die Ärzte als auch für die Apotheker das wichtigste Auswahlkriterium ihre Erfahrung mit den verschiedenen Produkten. Erst an zweiter Stelle steht die wissenschaftlich erwiesene Wirksamkeit. In punkto Evidenz unterschieden sich die Berufsgruppen allerdings: Am meisten Wert darauf legten die Apotheker (92\%) gegenüber 78\% der HNO-Ärzte und nur 67\% der Allgemeinmediziner. Der Preis hatte für 30\% der Ärzte und 20\% der Apotheker Relevanz. Bei den Anforderungen an die pharmakologischen Eigenschaften von Präparaten zur Therapie mikrobieller Erkrankungen lag die Wirksamkeit gegen Erreger auf Platz 1 (83\%), danach rangierten die Wirkung gegen Entzündung (76\%), gute Verträglichkeit (74\%) und die Wirksamkeit gegen Schmerzen (54\%). Die Art der Erreger spielt für die Auswahl eine eher geringe Rolle: $45 \%$ der HNO-Ärzte und nur 33\% der Allgemeinmediziner bzw. $10 \%$ der Apotheker hielten dies für die Medikationsempfehlung bei Schmerzen im Hals/Rachenbereich für wichtig.

\section{Antibiotische Therapie}

In der Mehrzahl der Fälle ist die akute Rhinosinusitis viral bedingt und somit eine antibiotische Behandlung nicht indiziert. Eine bakterielle Sinusitis stellt beim sonst Gesunden nur bei starken Beschwerden, Symptomzunahme im Verlauf, Fieber über $38,3^{\circ} \mathrm{C}$ oder drohenden Komplikationen eine Indikation zur antibiotischen Therapie dar. Bei Patienten mit schweren Grunderkrankungen oder Risikofaktoren wie chronisch entzündliche Lungenerkrankung, Immundefizienz oder Immunsuppression sollte ebenfalls ein Antibiotikum verschrieben werden.

Mittel der Wahl ist Amoxicillin. Bei chronischer Rhinosinusitis kann eine längere Kombinationstherapie mit Antibiotika und Steroiden als Operationsalternative in Betracht gezogen werden. Mittel der Wahl ist ein Aminopenicillin in Kombination mit einem Beta-Laktamasehemmer, alternativ aber auch ein Fluorchinolon. Die Therapie sollte nach Abklingen der Beschwerden noch über mindestens acht Tage fortgeführt werden. Bei akuten Exazerbationen einer chronischen Rhinosinusitis erfolgt die antibiotische Therapie wie bei der akuten Rhinosinusitis.

\section{Analgetische Therapie}

Bei akuter und chronischer Rhinosinusitis wird die Anwendung von rezeptfreien Analgetika wie Paracetamol, Diclofenac oder Ibuprofen nur bei Gesichts- und/ oder Kopfschmerzen empfohlen. Es gibt keine Hinweise für die Überlegenheit eines der Präparate. Zur Schmerzreduktion kann eine Akupunkturbehandlung versucht werden. Liegt eine Analgetika- 